\title{
Discovering the Human Heart, the Fall of Medical Science, and Correcting the Mistakes of Human Civilization
}

\author{
Emad Faiz Kamel \\ Date of birth 9july 1965 \\ Author of a book "the human heart is the basis of thinking, dreams during sleep" \\ A great scientific discovery in nature by Emad Faiz Kamel
}

\begin{abstract}
Before me there are only two men in all creation who did the cosmic phenomena analysis. The first is Galileo who discovered the earth is spherical the second is Isaac Newton who discovered the laws of gravitation. After that, God closed on the nature for three hundred years. So I am not a discoverer but I am an analyzer of cosmic phenomena. The heart affects the human brain with electromagnetic waves, and not, as medical science deceived us, the brain controls the heart with brain electricity. The sympathetic and parasympathetic nerves do not send the electricity from the brain to the heart but the sympathetic and parasympathetic nerves receive electromagnetic waves from the heart to the brain and all the body of human nature.
\end{abstract}

\section{INTRODUCTION}

Discovering the human heart, the fall of medical science, and Correcting the mistakes of human civilization. I am Emad Faiz from Egypt. I am sending you this scientific discovery, which is a analysis cosmic phenomena

Before me there are only two men in all creation who did the cosmic phenomena analysis. The first is Galileo who discovered the earth is spherical the second is Isaac Newton who discovered the laws of gravitation. After that, God closed on the nature for three hundred years. So I am not a discoverer but I am an analyzer of cosmic phenomena.

So I discovered that

The human heart is the basis of dreams which comes during sleep case and how I am able to make any person can dream while he is sleeping

> Human heart is the basis of any mental process (thinking, understanding, imagination and remembering.)

$>$ Human heart is the basis of movement

$>$ The heart is the basis of fixed mathematical equation to the pattern of human nature $\{$ F.E.W $(\mathrm{E} \mathrm{C} \mathrm{G})=1,1$ Or 1,2 H.Z / Sec \} = HN

$>$ The heart is the basis of human life or human death All these discoveries are made by physical laws and not by theories.
Therefore when death of the heart occurs with cardiac arrest because the heart has this system of physical laws that are the basis of human life so in this case the person will die the cause is not the stop of blood as claimed by medical science in the past but the cause is the stop of this physical system owned by the heart which is the basis of human life

This discovery is not only the science of medicine only, but combines three sciences

$>$ Firstly Medical Science

$>$ Secondly Science of Physics in Computer Engineering

$>$ Thirdly Science of Physics in Communication Engineering

\section{$>$ Firstly science of medicine}

We must to know that the heart has five sensory systems (five brains)

1-Sino Atrial Node

S-A-N

2-Atrio Ventricular Node

$\mathrm{A}-\mathrm{V}-\mathrm{N}$

3-Atrio Ventricular Bundle

A-V-B

4-Bundle Branch

B-B

5-Purkinje Fibers

$\mathrm{P}-\mathrm{F}$

These sensory systems produce waves of E.C.G (Electro cardio Gram)

1 - $\mathrm{P}$ wave Descriptive text that

2 - p r interval

3 - Q R S

4 - ST segment

5 - $\mathrm{T}$ wave

Secondly The geometries the physics $P C$ in the heart $C P$ $U$ (the heart is center processing unit)

It is well known that internet information, either wired or wireless, is only frequency, but not processing, but the processing and analysis are done by waves sent by the computer processor. Processor waves are measured by mega, mega is measured by hertz and it equals $10^{3} \%$ one thousandth of a second hertz, $\left(10^{3} / \mathrm{millisecond}\right)$. These are waves measured by hertz like the heart, These are waves measured by hertz like the heart, but in humans, it is limited to the E.C.G waves frequency speed, equals 1,1 or 1,2 hertz/sec.

This means that the heart is the CPU (central processing unit) 
Because the heart is the source of electromagnetic waves E.C.G and the frequencies of those waves equal to 1,1 or $1,2 \mathrm{Hertz} / \mathrm{Sec}$., in this case the heart is the CPU.

\section{Thirdly Geometries the communications physics in the nerves}

The sympathetic and parasympathetic nerves do not send the electricity from the brain to the heart until the heart works as the medical science claimed in the past but the heart works with its own mechanics.

But the electricity of the brain works to find magnetic field flux movement on the sympathetic and parasympathetic nerves as unit of measurement the scientist Orsted (Oe).

In this case, the sympathetic and parasympathetic nerves become an antenna wire according to the laws of physics in communications engineering and receive electromagnetic waves from the heart and send them to the brain and human body the sympathetic nerve sends the electromagnetic waves from the heart to the spinal nerve which control all the body and the parasympathetic nerve sends the electromagnetic waves from the heart to the brain.

In this case the brain becomes like TV screen appear the mental process but is not foundation of any mental process. The most important thing that changes the misunderstanding that prevailed in nature and medical science is that The sympathetic and parasympathetic nerves do not send the electricity from the brain to the heart but the sympathetic and parasympathetic nerves receive electromagnetic waves from the heart to the brain and all the body of human nature

I repeat this again until the nature the world and the medical science understand. The most important thing that changes the misunderstanding that prevailed in nature and medical science is that The sympathetic and parasympathetic nerves do not send the electricity from the brain to the heart but the sympathetic and parasympathetic nerves receive electromagnetic waves from the heart to the brain and all the body of human nature.

\section{A very important note;}

All human nerves are not electrical wire, meaning only electricity. Rather, they are an antenna wire carrying electromagnetic waves with electricity. This is why science and medicine have deceived nature and thought it was the brain. But in the beginning, we need to know how the sympathetic and parasympathetic turn into an antenna wires. Sympathetic and parasympathetic must be takes electric from the brain until is found the magnetic flux field movement.

In this case, they become two antennas wires of receiving the electromagnetic waves that come from the heart. These waves are coming the opposite of electrical coming from the brain. In this case the heart controls the brain and the human body with electromagnetic waves the medical sciences did not understand the role of the electromagnetic waves because these electromagnetic waves be worked by electric which comes brain

In the end, we must know that the nerves of the human do not received the electrical from the human brain only, but controlled by the frequencies of electromagnetic waves which comes from the heart but this waves be worked by the electric which comes from the brain.

The human brain in this case becomes a screen that is illustrated by mental processes but is not the basis of mental processes. But any mental processes comes from another physical system, the electromagnetic waves which comes from the heart.

- What is the use of the antenna wire according to the laws of physics in communications engineering?

The advantage of antenna wire is that it switches electromagnetic waves from the wireless state to the wire state

- What is the benefit of the sympathetic and the parasympathetic which takes electric from the brain?

The answer until the magnetic flux is found and become an antennas wire. In this case the sympathetic and the parasympathetic, they receive the electromagnetic waves that come from the heart and switches them from the path no nerves to the path nerves

\section{RESULT}

1- In this case the brain becomes like TV screen appear the mental process but is not foundation of any mental process. This is why science and medicine have deceived nature and thought it was the brain.

2- So I have to declare that any cardiologist in the world can get 6 pictures of those waves of E.C.G which come from the heart to limbs of the human body by device heart waves receiver and so any cardiologist does not know how those waves come from the heart to the limbs of the human body

3-we must know that the nerves of the human do not receive the electricity from the human brain only, but controlled by the frequencies of electromagnetic waves which come from the heart but this waves be worked by the electricity which comes from the brain.

First: The Experiences of dreams during sleep

How do dreams happen during sleep and what are dreams ?

I can give the fixed scientific method that makes any human being able to dream during sleep

Dreams happen in the following manner;

We have to divide the Intended sleep of the experiment subject into two periods. 
ISSN No:-2456-2165

\section{First Period}

1-The person who experiment should lay on his left side using one pillow

In this case, the heart pulse is affected, because the heart tilts slightly to the left which affects the heart sensory systems

2-While his head on the pillow, before to the case mode happen (sleep) he must prolonged the times of inhalation and the exhalation periods If the time of them in normal $2: 3 / \mathrm{Sec}$, he increases both of them to $6: 7 / \mathrm{Sec}$. in voluntary way This is the estimation method is not fixed. I emphasize the item.

However, he should keep this method until he falls asleep this is the main pillar because it is the basic for finding the experience I must emphasize the elongation of inspiration and exhalation in a voluntary way. he increases both to 6:7/to Sec.

It is not important to divide sleep duration into two equal periods. The first period might be quarter of an hour or more. afterwards he should be awakened by an alarm clock. however, he should not go back to sleep, but he is bound to fully wakeup, alert.

\section{Second Period}

When he returns to the second period

1-He must put another pillow, so that the heart pulse is not affected

2-he must sleep on the right side, so that In that position the heart is not affected by the pressure on it and its sensory systems too he is not required to do any changing respiration , like the first period, he must be with this case until return to sleep again

\section{The Result}

During the second sleep period, dreams happen naturally the person with the experience or the person who experimented will know that those dreams happened according to a scientific law, because dreams usually happen during that period of sleep and he will know the heart is the basis of the dreams.

\section{The Definition of dreams according to physics law}

Dreams are a change in the sensory systems of the heart S-A-N A-V-N A-V-B B-B P-F with few ways to this change must be in the first sleep time must be Sleep on the left side and increasing the time of the breath in and the time of exhalation together in a voluntary way using one pillow in the first sleep time in this the heart beat become slow.

Second sleep time you should sleep on the right side and return to normal breathing and do not do anything to breathe, using two pillows when the sleep in this case the heartbeats return to its normal.
In this case, the change in the Sensory devices of the heart be happened those sensory systems give us the waves of E.C.G, which affect the brain during the sleep by an electromagnetic waves and the speed of frequency is equals 1,1 or 1,2 hertz/sec according the speed of heart pulse

(This results in random television waves during sleep and this are the dreams)

\section{Second: How does thinking happen or any other process (understanding - remembering - imagination)?}

1 -The brain is connected to the heart by sympathetic and parasympathetic nerves

2-The heart affects the brain through E.C.G electromagnetic waves, the speed of which is 1,1 or $1,2 \mathrm{Hertz} / \mathrm{Sec}$. This means that the heart is the CPU (central processing unit) via the sympathetic and parasympathetic nerves in the manner of antenna wire

In that case, the human nerves do not only carry electricity from the brain, but electromagnetic wave frequency coming from the heart. I repeat this is the most important information in this discovery. When the brain becomes idle, electricity in the nerves becomes idles too. consequently, electromagnetic waves lose control over human nature although it still in the nerves but the electromagnetic waves pass in the nerves according to magnetism and not by movement of electrons of the material The effect of the electromagnetic waves is activated by electricity coming from the brain, including the effect on the brain itself, because it flows against the electricity in the sympathetic and parasympathetic nerves. this has led the medical sciences deceive that brain is the basis of thinking and all other mental processes, according to two cases the activity and the idle of brain electricity. medical sciences have failed to understand the role of electromagnetic waves coming from the heart, and it's the frequency speed of which is 1,1 or $1,2 \mathrm{Hertz} / \mathrm{Sec}$. and control the brain electricity. hence, the heart is a CPU (central processing unit)

Final

In the presence of electromagnetic waves in human nerves and through its interaction with electricity coming from brain, these electromagnetic waves interact with the eye movement in processing light to produce vision. It, also, interacts with the ear to produce hearing as well as its interaction with the mouth to produce speech. It provides magnetism to activate every movement in the body.

Therefore, it is not the electricity that comes from the cerebrum, but through the activation of electromagnetic waves coming from the heart.

And because they are electromagnetic waves, in this case they are processor waves, treating light falling on the eye, analyzing the electromagnetic waves coming to hearing, analyzing the sense of taste and smell with a biological process and analyzing touch through polarity, as they are the basis of speech movement, and because they are electromagnetic waves, they are physical laws. It is not a 
biological process from the human brain as medicine has deceived us

What are the mental processes? What is the mind?

It is a control of the heart on the brain by E.C.G wave frequency that is equal to 1,1 or $1,2 \mathrm{Hertz} / \mathrm{Sec}$.by the method of electromagnetic waves because in that case the heart is the CPU. my physics law proves all this. And according to the laws of physics in computer engineering.

1-Human direct this natural mental process, towards either thinking, imagination, remembering or understanding or any movement or any life

2-by this mental processes the dream happen through the sleep case

3-It is human life because any human without this mental processes he will die

After all, it is a natural process experienced by human nature and not as medicine claims that it is a biological process triggered by cerebrum centers.

According to the laws of physics in computer engineering. It is well known that internet information, either wired or wireless, is only frequency, but not processing, but the processing and analysis are done by waves sent by the computer processor. Processor waves are measured by mega, mega is measured by hertz and it equals $10^{3} /$ one thousandth of a second hertz, (10\%3illisecond). These are waves measured by hertz like the heart, but in humans, it is limited to the E.C.G waves frequency speed, equals 1,1 or $1,2 \mathrm{hertz} / \mathrm{sec}$.

The definition physical of thinking or any mental process (understanding - remembering - imagination). Thinking or any other mental process (understanding remembering - imagination) is analysis and processing of the electric wave of the brain through the frequency electromagnetic waves E.C.G coming from the heart, which affect the brain in the manner of electromagnetic waves, the speed of which equals 1,1 or 1,2 hertz/sec according as the speed of heart pulse.

\section{Third: Movement Laws}

Movement is caused by the heart that gives the human nature magnetic is coming from heart interacting by electricity coming from cerebrum. The human nerves do not have electricity from cerebrum only but also has electromagnetic waves frequencies coming from the heart. Again, this is the most important piece of information in the discovery in brief the movement is the activating the electromagnetic waves which come from the heart through the electricity which comes from the brain all this is taking place on all the nerves of the human nature so when the electricity which comes from the brain becomes inactive, also the electromagnetic waves which come from the heart become inactive.

\section{The mistakes found in medical science}

\section{First Evidence}

According to the laws of movement, it is divided into magnetic or mechanical. Medical sciences have committed a mistake when it concentrated on electricity only to produce movement in human body, but electricity does not produce movement solely, but the magnetic is basis. The same applies, for example, to the fan, which does not produce movement through electricity solely, but the magnetic is basis. We should know that magnetism in the human body comes from the polarity produced by the heart

\section{Second Evidence}

\section{The relationship between the mental process coming from the heart and the movement}

After we know the effect of the heart on the brain and also the effect of the heart on the whole body by the controlling of the electromagnetic waves coming from the heart on all the nerves of the human .

Now come to understand all the movements of the human body and how it comes from the mental process which coming from the heart.

If we follow the science of medicine which dropped and deceived nature of humanity in the largest theory of error, the science of medicine deceived and dropped the nature of humanity and told us that the brain controls the human body by the electricity coming from the brain and these are the biggest errors of medicine that dropped the human nature in the largest theory of astray did not know the science of medicine the role of electromagnetic waves coming from the heart.

1 - How these waves control the nerves of the human body 2 - the role of these waves as a physic laws in the human life Here is one example that illustrates all the wrongs in medical science.

The example is

When I write the word Britain, the science of medicine comes and allege us this movement comes from the electricity that comes from the human brain and thus controls the nerves and thus controls the human body and ultimately controls human movement in the hand.

When I write the word the queen Elizabeth the science of medicine deceive us and allege to us the same deceit that she said when I wrote the word Britain, here is the greatest misfortune that medicine deceived the nature, whom I shall mention in a number of innumerable points.

1-What kind of electricity which comes from the brain has methods and photos are physic different

2-How do the brain biologically makes the electricity physic differently which comes from the beginning

3 - How this electricity physic goes in the nerves by biological method together in different methods 
Therefore, after we knew that it is impossible and very impossible in all kinds of natural sciences and in all kinds of scientific logic to be the brain is the process of mental therapy is not by the way of biology and the way of physic electricity and more that there is no the method we can understand how the brain do so far because nature fell in The most dangerous misguide when it was claimed by the medicine logic that the brain is the basis of mental processes But after we knew the role of the heart.

1-It is the source of electromagnetic waves and that these waves control the brain through the nerve of the parasympathetic and on the human body through the nerve of the sympathetic and how affect the spinal nerve

2-not only this but the effect here is not the control and biological effect, but the physic effect of electromagnetic waves at a frequency of equal to1.1 Or $1.2 \mathrm{~Hz} /$ second

Here we make sure scientifically that the heart is the basis of mental processes and the basis of mathematical fixed equations to the human nature and the basis of all life.

We must make sure, in a scientific and physic sense that it is the heart and that after I have done the greatest analysis and scientific discoveries of physics to this phenomenon of cosmic universality, so If it lived the nature without analyzing this cosmic phenomenon the obscures ambiguity and ignorance stay control the nature the science and the life in this case.

How is the relationship between the electricity coming from the brain and electromagnetic waves which comes from the heart ?

The electrical which comes from the brain is working the electromagnetic waves which coming from the heart, but the electricity coming from the brain is not the electromagnetic waves which is the basis of movement and the mental processing which comes from the heart .

Such as mobile and television that electricity is working the mobile and the television, but electricity is not the electromagnetic waves, which is the means of communication in mobile and television.

Also, if the human brain stops, in this case, the electrical, physical, or active unit stop the working of the electromagnetic waves coming from the heart but the death is not happen but if the heart stops, it will stops the physic unit of the electromagnetic waves which is the basis of life and movement and mental processes and the basis of equations fixed mathematically of the pattern human nature.

In this case death occurs and life ends The basis of the biggest mystery in nature is dreams during sleep. The most important observation in this discovery whom illustrates the relationship between the electricity which comes from the brain and electromagnetic waves which comes from the heart.
1-The electricity that comes from the brain passes in the nerves, while the electromagnetic waves that come from the heart pass on the nerves and so the electromagnetic waves of the heart moves on the nerves in direction of electricity or reverse the direction of electricity that comes from the brain

2-This is the main reason why the electricity which comes from the brain powers the electromagnetic waves which comes from the heart. Because the electricity which come from the brain gives the energy for the electromagnetic waves which come from the heart by the magnetic flux whom is found in the electricity

3-The electricity that comes from the brain is the operating energy to the electromagnetic waves that comes from the heart.

4-But the energy of the electromagnetic waves of the heart control of nerves in final because it is a magnetic energy movement.

5-Ultimately, however, the electromagnetic waves are entirely in control on the nerves because they possess magnetic power so it is a magnetic energy movement.

Finaly

Electromagnetic Waves which comes from the heart is the center processing unit of the human mental. the movement the life the basis of equations fixed mathematically of the pattern human nature.

The basis of the biggest mystery in nature is dreams during sleep. From the beginning it takes shape in the woman's womb.

The main idea of this discovery is based on the fact that the human heart controls and affects the cerebrum through by electromagnetic waves.

I has a specific law in my name, owner of this discovery. I construed the basis of this law through the physics relationship between the process from Radio and Television Building electromagnetic transmission and the process of reception of this antennas electromagnetic waves.

Therefore the truth is, life, mental processes, mathematical fixed equations to the pattern of human nature, laws of motion and the dreams phenomenon are the result of the magnetic polarity which come from the human heart, which is activated by the human cerebrum electricity.

In human life electromagnetic waves begin first before electricity. After the cellular configuration comes the composition of the allocated cells. So the heart will form in the fourth week and give electromagnetic waves in the fifth week. The formation of brain cells begins in the 12th week. Here is the question of how the heart works itself from the fifth week until the twelfth week alone without the presence of the brain. This has led the sciences of medicine concentrate on the human cerebrum electricity, while ignoring how to explain the magnetism which come from the human heart. The Fourth Fixed Mathematical Equation 
Firstly

The geometries the physics in the heart (C P U ). The heart is center processing unite. The patterns of objects The patterns of objects differ from each other not because of physiological composition which is certainly similar among all these organisms but the patterns of objects differ from each other because of the different laws of physics Because each object has a physics system which different from a system the other object The mathematical fixed equation to the pattern of human nature, (the mental processes and this is the mind of human nature ). It is the control from the heart on the cerebrum by waves frequencies E.C.G of the human heart which equal to 1,1 or $1,2 \mathrm{Hertz} / \mathrm{Sec}$., because in that case the heart is the CPU.

All my laws physics previously mentioned proved all this Humans nature direct this natural mental process according as thy will towards either thinking, imagination, remembering or understanding and this is a natural process experienced by human nature and not as medicine claims that it is a biological process comes by cerebrum centers in final this are the mental processes and this is the mind of human nature.

\section{Like Computers}

According to the laws of physics in computer engineering. It is well known that internet information, either wired or wireless, is only waves frequencies, but not processing, but the processing and analysis are done by waves sent by the computer processor. Processor waves are measured by mega, mega is measured by hertz and it equals 103/one thousandth of a second hertz, (103/millisecond). These are waves measured by hertz like the heart, but in humans, it is limited to the E.C.G waves frequency speed, equals 1,1 or 1,2 hertz/sec

secondly

Geometries the communications physics in the nerves the sympathetic and parasympathetic nerves do not send electric from the brain to makes the heart works as the medicine science claimed that in the past but the heart works with its own mechanics. In this case, the sympathetic and parasympathetic nerves become an antenna wire according to the laws of physics in communications engineering that receive electromagnetic waves from the heart and send them to the brain and human body the sympathetic nerve sends the electromagnetic waves from the heart to the spinal nerve which control all the body and the parasympathetic nerve that sends the electromagnetic waves from the heart to the brain.

But in the beginning, we need to know how the sympathetic and parasympathetic turn into an antenna wires ?

Sympathetic and parasympathetic nerves must be takes electric from the brain until the magnetic flux is found In this case, they become two antenna wires of receiving the electromagnetic waves that come from the heart. these waves are coming the opposite of electrical which coming from the brain at the nerves.
In this case, the heart becomes a source of electromagnetic waves for the brain and body and not an organ that receives electricity from the brain In this case, the heart controls the brain and the human body with electromagnetic waves. The medical sciences did not understand the role of the electromagnetic waves because these electromagnetic waves be worked by electric which comes brain.

In the end, we must know this all the nerves of the human body do not received the electrical from the human brain only, but controlled by the frequencies of electromagnetic waves which comes from the heart but this waves be worked by the electric which comes from the brain

I repeat this again

In this case, the heart becomes a source of electromagnetic waves for the brain and body and not an organ that receives electricity from the brain.

In this case, the heart controls the brain and the human body with electromagnetic waves. The medical sciences did not understand the role of the electromagnetic waves because these electromagnetic waves be worked by electric which comes brain.

we must know this all the nerves of the human body do not received the electrical from the human brain only, but controlled by frequencies of electromagnetic waves which comes from the heart but this waves be worked by the electric which comes from the brain.

So I have to declare that any cardiologist in the world can get 6 pictures of those waves of E.C.G and he does not knows how they come from the heart to the limbs by (Device Heart waves receiver).

The human brain in this case becomes a screen that is illustrated by mental processes but is not the basis of mental processes any mental processes comes from the waves of the heart.

What is the use of the antenna wire according to the laws of physics in communications engineering?

by the antenna wire the electromagnetic waves are switched from the wireless to the wire what is the benefit of the sympathetic and the parasympathetic until takes electric from the brain?

Until the magnetic flux field is found in sympathetic and parasympathetic nerves and whom it becomes an antennas wire

In this case the sympathetic and the parasympathetic nerves, takes the electromagnetic waves which come from the heart and turn them from the method no nerves to the method nerves

The sympathetic and parasympathetic nerves become an antenna wire according to the laws of physics in communications engineering that receive electromagnetic 
waves from the heart and send them to the brain and all the nerves human body

\section{HOW THE HEART IS THE BASIS MATHEMATICAL FIXED EQUATION TO THE PATTERN OF HUMAN NATURE?}

F.E.W $(\mathrm{E} \mathrm{C} \mathrm{G})=1,1$ or 1,2 H.Z / Sec=HU

These pulses and movement coming from the heart are representing the life and nature of man is not through the pumping of blood or it is a pump works by the electrically coming from the brain as deceived us by medical science which deceived human nature too.

But these pulses and movements coming from the heart is the basis of the pattern of human nature with it is system of physical laws.

We must understand the following facts about the human heart.

1-The heart is the source of electromagnetic waves coming from five brain types found in the human heart.

(S-A-N A-V-N A-V-B B-B P-F, )

and this waves are (Electro Cardio Gram )

1-P wave

2- $\mathrm{p}$ r interval

3-Q R S

4-ST segment

5-T wave

These waves have a frequency speed is equals 1,1 or 1,2 Hertz/Sec.

2 -The electricity that comes from the brain on the nerves is the only physics way that makes the nerves receive the electromagnetic waves which comes from the heart and walk on the nerves and control the nerves too.

I repeat this item once again.

2 -The electricity that comes from the brain in the nerves is the only physics way that makes the nerves receive the electromagnetic waves which comes from the heart and walk on the nerves and control the nerves too.

Therefore, if the electricity coming from the brain that passes in the nerves is stopped in this case, the activation of the electromagnetic waves coming from the heart, which also travels on the nerves, will be stopped by the brain electricity.

Because this electromagnetic waves of the heart moves on the nerves in direction of electricity or reverse the direction of electricity that comes from the brain.

I'll write you through the mathematical fixed equation which determine the pattern the nature of the human.

I will write to you how I can overthrow the Darwin 's theory.
F.E.W ( E C G ) = 1,1 0r 1,2 H.Z / Sec=HU

F.E.W = Frequencies of electromagnetic waves

E C G = Electro cardio Gram

1,1 or $1.2=$ Fixed arithmetic number

$\mathrm{H} . \mathrm{Z}=$ Gauge $\mathrm{Hz}$ oscillations or $\mathrm{Hz}$ frequency

$\mathrm{Sec}=$ Unit of time measurement in seconds

1 - Women differ from men according to physiological composition

2 - Women differ from men according to cellular composition

3 - Women differ from men according to genetic composition

But women and men are similar in a fixed mathematical equation which determines the pattern of human nature. Because the fixed mathematical equation is based on a physical law that determines the pattern of human nature and not the physiological composition and not the cellular composition and not genetic composition so the human origin did not come from monkey and does not turn into another being.

\section{THE ARTIFICIAL HEART}

Some medical societies as well as concepts announce that the heart is a pump only this is deception to human nature, which underlies the probability of replacing a normal heart by an artificial pump. I repeat here that the mistakes are based on theories and not scientific laws but what uncovers the issue is respiration. According to the laws physics of movement, movement is divided into either magnetic or mechanical movement. However, medical sciences have committed a mistake when concentrated on electricity only as a source of movement, or origin of movement in the human body. However, According to the laws physics electricity does not generate movement on its own; but there should be magnetism with electricity as well. We should know that magnetism in the body comes from the polarity produced by the heart.

The magnetism that comes from the heart here does not come in a magnetic field way, but it comes in an electromagnetic waves way whom pass on and control the nerves by brain electricity should know that if the brain halts, only electrical paralysis occurs, but the heart and respiration do not stop. If the heart stops, the body movement stops completely, including respiration. Respiration does not stop because of brain idleness or its subjection to anesthetic but it stops in one case only, which is absence of heart pulse. Respiration returns when the heart pulses again, and medical sciences know this well. Respiration is, therefore, a magnetic polarized movement that comes from the heart through the sympathetic nerve, which comes from the fourth paragraph located in the neck which is the only direct link between the heart and respiration ( cardiac pulmonary plexuses ). Medical sciences should not convince us the idea, based on electricity only and ignore the role of magnetism coming from the heart. 
Therefore, when we are told that there is a so-called artificial heart that can replace the natural heart, it would be a huge mistake against nature. How can the artificial heart give us the magnetic polarization, which is produced by the normal heart through the frequency that reaches the farthest areas of the body, which are the limbs that any cardiologist in the world take 6 pictures of E.C.G waves, but any cardiologist does not know how those waves pass from the heart to the limbs by the nerves too. The Cases of fainting because of heart feebleness means the weakness of electromagnetism coming from the heart, which leads to stopping any movement; hence fainting. Not just fainting but also death because the electromagnetic waves which comes from the heart are the basis of the pattern and nature of human life.

Therefore, there is nothing called artificial heart to substitute the natural heart one;

1-There is an artificial heart that is used during the surgery to keep blood circulation flowing so that blood does not clot inside the blood vessels

2- There might be an artificial heart that helps in providing the natural heart muscle with electric pulse that is useful in systole and diastole.

But there is no complete artificial heart that can substitute a natural heart. For this reason, there is a natural heart Transplantation to replace another natural heart.

But there is no complete artificial heart transplantation to replace a natural heart completely. This is a warning to some international corporations which imagine that the artificial heart substitute for the complete natural heart .

Warning in heart transplants

1 - The heart of the pig is not suitable to be a substitute for the human heart because it gives the electromagnetic waves that determine the pattern of nature of an animal and not a human.

This was explained in the previous article in the heart, the basis of the mathematical constant equation that determines the pattern of human nature.

( F.E.W $($ E C G ) = 1,1 0r 1,2 H.Z / Sec=HU )

2 - The artificial heart is not a substitute for the natural heart because it does not give the electromagnetic waves.

3 - The natural heart only it is the substitute for the natural heart because it gives electromagnetic waves determine the pattern of nature of the human.

\section{Latest notes}

Brain electricity reduces the heartbeat, not as medical science claims that brain electricity occupies the heartbeat.

I am surprised but I am shocked by theories of false medicine.

Sometimes it claims that the brain's electricity makes the heart beat and sometimes it claims that if the VEGAS nerve is active, it stops the heart.
Question: Does brain electricity makes the heart beat or makes the heart stop?

But the heart works with its own mechanism by speed from 105 to 120 beats per minute.

But the sympathetic and parasympathetic nerve becomes an antenna wire according to the laws of physics in communications engineering

By electricity coming from the human brain in them are magnetizing part from the magnetic of heart this reduces the heartbeat from speed 105 or 120 to 70 or 80 pulse per minute.

Therefore, if a brain's electricity is interrupted or stopped, in this case the heartbeats will become from 70 or 80 to 105 or 120 or return to 105 or 120

Finally, the three physical laws that underlie my analysis of this cosmic phenomenon.

The first

The scientific law of physics which is the basis of my scientific discovery 1 -

If electric current passes through a conducting wire between two ends, from $\mathrm{A}$ to $\mathrm{B}$, this current tries to find what is called, "to find magnetic field flux movement" through the movement of matter electrons in the wire, which results in the following;

1-End $\mathrm{A}$ has the potential of sending electromagnetic wave frequency to end $\mathrm{B}$, which flows with the electric current. It is described as the passing of electromagnetic wave frequency from the static medium to the vibrant medium, which is similar to television transmission.

2-End $\mathrm{B}$ has the potential to receive electromagnetic wave frequency and sends it to end A against the electric current. It is described as passing the electromagnetic waves from the vibrant medium to the static one, which is similar to antenna receipt of television to transmission frequency.

The basis of the mathematical fixed equation. The control by the human heart on the human nature (the brain and the whole human body) by an electromagnetic waves frequency equal to 1.1 or $1,2 \mathrm{hertz} / \mathrm{sec}$ according on the speed of heart

\section{The second}

2-The Definition of dreams according to physics law

Dreams are a change in the sensory systems of the heart $S$ A-N A-V-N A-V-B B-B P-F, with few ways to this change must be

1-in the First sleep time must be Sleep on the left side and increasing the time of the Inhalation and the time of exhalation together in a voluntary way using one pillow in the first sleep time in this the heart beat become slow

2-Second sleep time you should sleep on the right side and return to normal breathing and do not do anything to breathe, using two pillows when the sleep in this case the heartbeats return to its normal 
In this case, the change in the Sensory devices of the heart be happened those sensory systems give us the waves of E.C.G, which affect the brain during the sleep by an electromagnetic waves and the speed of frequency is equals 1,1 or 1,2 hertz/sec according the speed of heart pulse This results in random television waves during sleep (dreams)

The third

3-The Definition of thinking or any mental process (understanding- remembering - imagination) according to physics law.

Thinking or any other mental process (understanding remembering - imagination) is Analysis and processing of the electric wave of the cerebrum through E.C.G frequency waves Coming from the heart, which affect the cerebrum in the manner of electromagnetic waves the speed of frequency equals 1,1 or 1,2 hertz/sec according the speed of heart pulse

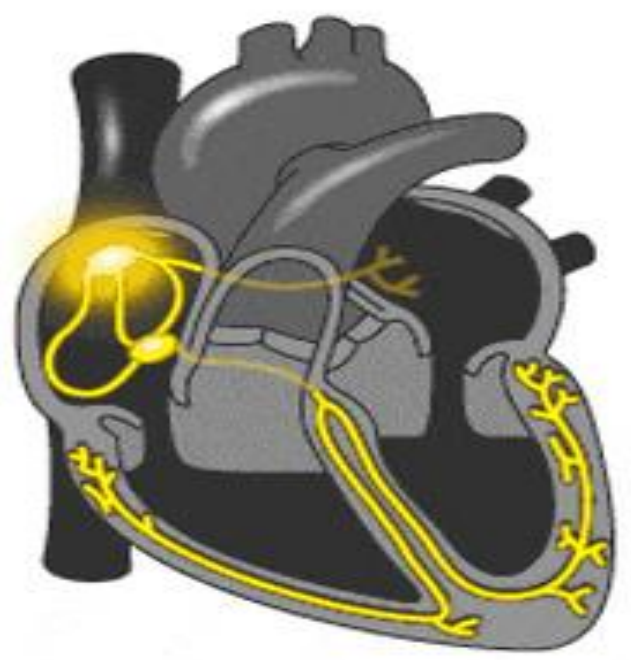

Fig 1

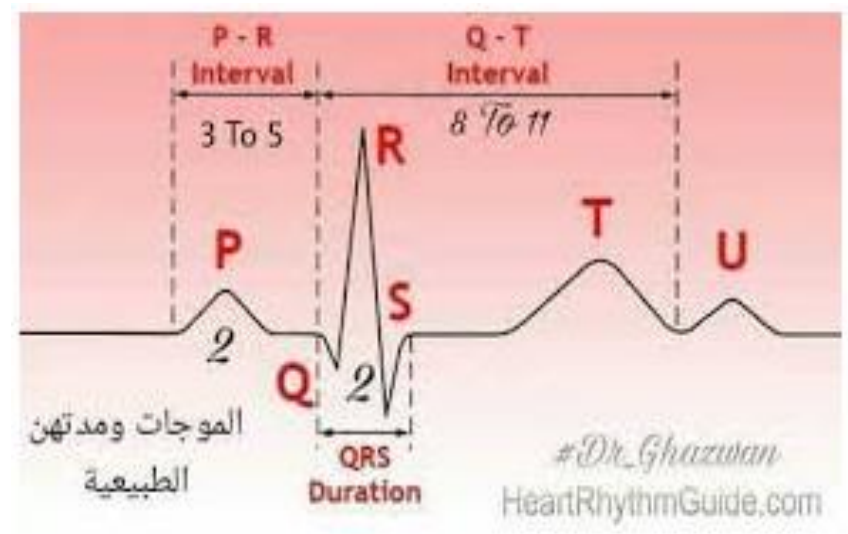

Fig 2

This picture is from the internet It shows the heart as a physical system, not as the medicine sciences deceive the nature in the past

\section{THE EDITORIAL STAFF}

The truth is not taken in forms or molds but the truth is taken its essence. Please accept this truth, not in the form of an article written, but as a fixed fact that corrects the errors of nature.

https://www.youtube.com/watch?v=CT98Y9vGCSE

The truth is that seeing and hearing is better than reading this video site on YouTube.

\section{REFERENCES}

[1]. Dr Zaki, Ashraf. "Basic Electrocardiogram" 200422043 record and books publishing house. Cairo, Egypt

[2]. studies on transmission and aerial reception ( antenna wire from the Internet. https://en.wikipedia.org/wiki/Television_antenna

[3]. Course of studies for two years on computer science and teaching IBI institute in Egypt.

[4]. Dr Muhammad Ali, Fadil "treatment without medications" 2006 edition, a new Egyptian theory

[5]. Surgeon el Haj, Salim "heart transplanting operation from human to human". https://www.youtube.com/watch?v=cbqVaRYR3vs

[6]. All pictures about the human heart and Electromagnetic waves are taken from the Internet . https:/www.facebook.com/2105307939751622/posts/ 2287017364914011/

[7]. Dr. Abdel El Hadi, shareef the former director of the national institute for the heart, in Egypt supervised and revised my discovery and attended all my lectures about the human heart, also he wrote the introduction for mu book

[8]. Studies that explain the neurological relationship between the heart and the lung (Breathing) https://www.youtube.com/watch?v=aqt1 VgtoJ7Y\&t=6 $57 \mathrm{~s}$ 